\title{
Photoreceptor responses of fruitflies with normal and reduced arrestin content studied by simultaneous measurements of visual pigment fluorescence and ERG
}

\author{
Gregor Belušič • Primož Pirih • Doekele G. Stavenga
}

Received: 6 July 2009/Revised: 1 November 2009/Accepted: 3 November 2009/Published online: 19 November 2009

(C) The Author(s) 2009. This article is published with open access at Springerlink.com

\begin{abstract}
We have simultaneously measured the electroretinogram (ERG) and the metarhodopsin content via fluorescence in white-eyed, wild-type Drosophila and the arrestin2 hypomorphic mutant $\left(w^{-} ; \operatorname{arr} 2^{3}\right)$ at a range of stimulus wavelengths and intensities. Photoreceptor response amplitude and termination (transition between full repolarization and prolonged depolarizing afterpotential, PDA) were related to visual pigment conversions and arrestin concentration. The data were implemented in a kinetic model of the rhodopsin-arrestin cycle, allowing us to estimate the active metarhodopsin concentration as a function of effective light intensity and arrestin concentration. Arrestin reduction in the mutant modestly increased the light sensitivity and decreased the photoreceptor dynamic range. Compared to the wild type, in the mutant the transition between full repolarization and PDA occurred at a lower metarhodopsin fraction and was more abrupt. We developed a steady-state stochastic model to interpret the dependence of the PDA on effective light intensity and arrestin content and to help deduce the arrestin to rhodopsin ratio from the sensitivity and PDA data. The feasibility of different experimental methods for the estimation of arrestin content from ERG and PDA is discussed.
\end{abstract}

\footnotetext{
G. Belušič $(\bowtie)$

Department of Biology, Biotechnical Faculty, University of Ljubljana, Večna pot 111, 1000 Ljubljana, Slovenia

e-mail: gregor.belusic@bf.uni-lj.si

P. Pirih - D. G. Stavenga

Department of Neurobiophysics, University of Groningen,

Nijenborgh 4, 9747 AG Groningen, The Netherlands
}

Keywords Drosophila photoreceptor - Arrestin . PDA $\cdot$ ERG $\cdot$ Metarhodopsin fluorescence

\section{Introduction}

The phototransduction process of animal photoreceptors starts with the absorption of light by visual pigment molecules, which causes conversion of the native rhodopsin state into an active metarhodopsin state, resulting, after several biochemical steps, in a change in the membrane potential of the photoreceptor (Minke and Hardie 2000; Hardie and Raghu 2001). The temporal resolution of the visual process depends on the speed of the biochemical chain and on the lifetime of the active metarhodopsin state, the trigger of the phototransduction chain. Inactivation of the metarhodopsin state occurs on binding of an arrestin molecule. The arrestin binding constant and its concentration thus are crucial factors in determining the temporal resolution, as well as the time course and extent of light adaptation of the photoreceptor (Hardie 2001).

The metarhodopsin state of vertebrate visual pigments is thermolabile, resulting in degradation of the metarhodopsin molecules and arrestin release. The metarhodopsin state of invertebrate visual pigments is, however, thermostable. This has led to the development of a different strategy of visual maintenance, as has become clear from extensive studies on the main photoreceptor class of the fruitfly, Drosophila, the R1-6 photoreceptors. On photon absorption, their rhodopsin (Rh1), which absorbs maximally in the blue-green, converts into a metarhodopsin state that absorbs maximally in the orange wavelength range (Ostroy et al. 1974; Salcedo et al. 1999). Absorption of a photon by the metarhodopsin causes the reverse process, namely conversion of the metarhodopsin into the rhodopsin state. 
In Drosophila and other higher dipterans, the conversion direction from metarhodopsin to rhodopsin is under normal light conditions augmented by the red screening pigment (Stavenga 2002). Photoconversion of the metarhodopsin is followed by arrestin release, after which the resulting native rhodopsin is ready for another round of phototransduction (Byk et al. 1993; for a recent review, see Hardie and Postma 2008).

If exposed to bright monochromatic blue light for a sufficiently long period, fruitfly R1-6 photoreceptors remain depolarized in the dark for hours (Cosens and Briscoe 1972; Minke et al. 1975). The persisting electrophysiological signal, a special phenomenon in the phototransduction process of Drosophila, has been named the prolonged depolarizing afterpotential (PDA). More recent studies on fruitfly mutants have clarified this finding: bright blue light creates more metarhodopsin molecules than can be blocked by the available arrestin, leaving a surplus of active metarhodopsins that keep the phototransduction process going, even in complete darkness (Byk et al. 1993; Dolph et al. 1993). A PDA is readily created in white-eyed fruitflies, which lack the blue light-absorbing screening pigments, but it is realized also in red-eyed flies if shortwavelength light of sufficient intensity and duration is applied (Lo and Pak 1981).

A quantitative understanding of the phototransduction process and its dependence on arrestin is strongly facilitated by the use of Drosophila mutants. Drosophila photoreceptors express two arrestin forms, arrestin1 (Arr1) and arrestin2 (Arr2), originally identified as phosrestin 2 and 1, respectively (Matsumoto and Yamada 1991). Arrestin2 is approximately sevenfold more abundantly expressed than arrestin1 in the wild type. Arrestin2 is the functionally more important form for the inactivation of metarhodopsin (Dolph et al. 1993). Compared to the wild type, arr2 mutants have a log unit lower threshold for entering the PDA state (Dolph et al. 1993; Vinós et al. 1997; rev. Hardie and Postma 2008). The residual arrestin in $w^{-} ; \operatorname{arr}^{3}$ is mostly arrestin 1 . In the absence of arrestin2, arrestin 1 can inactivate metarhodopsin, albeit less effectively, resulting in a slow course of inactivation in the $\operatorname{arr} 2^{3}$ mutant (time constant of current inactivation: wild type, $\tau=20 \mathrm{~ms}$, arr $^{3}$ mutant, $\tau=167 \mathrm{~ms}$; Ranganathan and Stevens 1995). Using the $\operatorname{arrl} l^{l}$ mutant, the absence of arrestin1 has not been shown to confer a deactivation defect. The role of arrestin1 in metarhodopsin inactivation has been shown indirectly, through a severe deactivation defect in the double $\operatorname{arr} 1^{1} ; a r r 2^{3}$ mutant (Dolph et al. 1993). A recent study by Satoh and Ready (2005) attributed an important intracellular trafficking role to arrestin1.

Arrestin2 translocates on the subcellular level between the microvilli and the soma in a light-dependent manner (Lee et al. 2003). The assumption that its concentration in the subcellular compartment is variable and regulated raises several questions: for instance, what is the adequate arrestin concentration in wild-type flies under normal environmental conditions, and how does the arrestin concentration determine the temporal resolution of fruitfly photoreceptors? Here, we analyze the role of arrestin by comparing the electrical responses of white-eyed fruitflies $\left(w^{-}\right)$, where the photoreceptors have the normal, wild-type phototransduction components, with the responses of white-eyed hypomorphic $\operatorname{arr} 2^{3}$ mutants $\left(w^{-} ; a r r 2^{3}\right)$, where the photoreceptors have a reduced arrestin 2 content. We specifically study the transition to the PDA state in relationship with visual pigment conversions, which were measured with the aid of metarhodopsin fluorescence.

\section{Materials and methods}

\section{Experimental animals}

Experiments were performed on two strains of fruitflies (white-eyed wild type, $w^{-}$; and white-eyed arrestin2 mutant, $w^{-} ; a r r 2^{3}$ ), donated by the Biosensorics Group of the University of Hohenheim, Germany. The wild-type flies were kept on a 12/12-h light/dark cycle, while the arrestin mutants were kept in constant darkness, to avoid retinal degeneration. The flies were reared on a standard cornmeal diet at room temperature $\left(20^{\circ} \mathrm{C}\right)$. The experiments were performed on 5 days old wild-type flies and on 1-day-old arrestin mutants at room temperature.

\section{Preparation}

The flies were immobilized with beeswax, fixed to a plastic pipette tip and positioned in a miniature goniometer. A chloridized silver wire (diameter $0.05 \mathrm{~mm}$ ), inserted into the thorax of the fly, served as the reference electrode. After checking the viability of the preparation by inspecting the dark trapezoidal pseudopupil under a dissecting stereomicroscope, the preparation was transferred to the stage of the microscope in the setup. The recording electrode was inserted horizontally from the side of the microscope stage, just beneath the cornea, under visual control using a low power objective.

\section{Optical and electrophysiological setup}

The experimental setup for the light stimulation of the eye and concurrent measurements of the fluorescence and the electroretinogram (ERG) was built around a Leitz Orthoplan epi-illumination microscope. The objective was a $20 \times$ LWD, NA0.40 (Olympus, Japan). The light stimulator consisted of a $75 \mathrm{~W}$ XBO lamp, a shutter and a 
monochromator (1/8 m, Newport Oriel, USA) with a bandpass (FWHM) of about $15 \mathrm{~nm}$. The measurement path for the fluorescence recordings consisted of an adjustable field diaphragm, a red long-pass filter $(>695 \mathrm{~nm})$ and a photomultiplier (PMT; R928, Hamamatsu, Japan). The PMT signal was low-pass filtered at $10 \mathrm{~Hz}$. The field diaphragm in the observation path was stepped down and positioned to coincide with the image of the deep-pseudopupil (DPP), to optimize the fluorescence signal to background ratio. For ERG measurements, we used a DAM-50 amplifier (WPI, USA) as the headstage. The amplifier was operated in single-ended DC mode with $10 \times$ gain. The signal was further amplified and low-pass filtered at $100 \mathrm{~Hz}$ with an AM502 plug-in amplifier (Tektronix, USA). For the electrophysiological recordings, we used borosilicate glass microelectrodes pulled from capillaries (OD $1.5 \mathrm{~mm}$, ID $0.7 \mathrm{~mm}$, with filament) on a P97 puller (Sutter, USA). The electrodes were filled with Insect Ringer and had a resistance of $<3 \mathrm{M} \Omega$. The ERG magnitude at saturating light intensities was about $20 \mathrm{mV}$.

Stimulation protocol, acquisition and data analysis

The ERG and PMT signals were sampled at $1 \mathrm{kHz}$ by a CED1401plus laboratory interface, operated under the control of the software package WinWCP (Strathclyde Electrophysiology software version 4.0.5; Dempster 2001), which also controlled the shutters and the monochromator. First stage off-line analysis (measurements of ERG magnitudes and fitting of fluorescence decay exponentials to the PMT signals) was performed in the waveform measurement and curve fitting module of WinWCP. The fitted parameters were then transferred to Prism 4.0 (GraphPad, USA), where further analysis was performed.

Visual pigment photochemistry and metarhodopsin fluorescence measurements

The main visual pigment of Drosophila (Rh1) has two thermostable states, rhodopsin (R) and metarhodopsin (M), which are photointerconvertible. The photochemistry of fruitfly visual pigment, therefore, is described by the scheme $\mathrm{R} \leftrightarrow \mathrm{M}$, with rate constants $k_{\mathrm{R}}$ and $k_{\mathrm{M}}$ for the photoconversions of $\mathrm{R}$ to $\mathrm{M}$ and of $\mathrm{M}$ to $\mathrm{R}$, respectively (see e.g., Stavenga and Schwemer 1984). The rate constants are $k_{\mathrm{R}}=\beta_{\mathrm{R}} I$ and $k_{\mathrm{M}}=\beta_{\mathrm{M}} I$, where $I$ is the light intensity, and $\beta_{\mathrm{R}}$ and $\beta_{\mathrm{M}}$ are the photosensitivities of the $\mathrm{R}$ and $\mathrm{M}$ state, respectively. For the photosensitivity $\beta=\gamma \alpha$ holds, where $\gamma$ is the quantum efficiency for photoconversion and $\alpha$ is the molecular absorption coefficient. The quantum efficiencies are probably wavelength independent for both the R and M state; photosensitivity and absorption spectra are then proportional. The molecular absorption coefficient and quantum efficiency of fruitfly visual pigment is unknown, but presumably they are similar to the values known for bovine rhodopsin, for which at the peak wavelength $(498 \mathrm{~nm}) \quad \alpha_{\max }=1.56 \times 10^{-16} \mathrm{~cm}^{2}$, and $\gamma=0.65$, yielding a photosensitivity of $\beta=0.01 \mathrm{~nm}^{2}$ (Dartnall 1972; Kim et al. 2001). Illumination of bovine rhodopsin causes photoconversion and bleaching with an exponential time course, with time constant $\tau=1 /(\beta I)$, meaning that a light flux of $10^{16}$ photons $\mathrm{cm}^{-2} \mathrm{~s}^{-1}$ (at the peak wavelength) results in a time constant of $1 \mathrm{~s}$.

When a population of Drosophila visual pigment molecules, initially in the rhodopsin state, is irradiated by monochromatic light, the time course of the photoconversion process is described by:

$f_{\mathrm{M}}(t)=f_{\mathrm{M}}(\infty)[1-\exp (-t / \tau)]$

where $f_{\mathrm{M}}(t)$ is the fraction of molecules in the metarhodopsin state at time $t ; f_{\mathrm{M}}(\infty)$, the fraction of metarhodopsin molecules in the photosteady state, at $t=\infty$, is a function of the ratio of the photosensitivities of $\mathrm{R}$ and $\mathrm{M}$ :

$$
f_{\mathrm{M}}(\infty)=k_{\mathrm{R}} /\left(k_{\mathrm{R}} / k_{\mathrm{M}}\right)=\beta_{\mathrm{R}}\left(\beta_{\mathrm{R}}+\beta_{\mathrm{M}}\right)=\left(1+\beta_{\mathrm{M}} / \beta_{\mathrm{R}}\right)^{-1} .
$$

The rhodopsin and metarhodopsin of the main visual pigment of Drosophila (Rh1) absorb maximally at 486 and $566 \mathrm{~nm}$, respectively (Fig. 1a; Salcedo et al. 1999). At the isosbestic wavelength, $\lambda_{\text {iso }}=505 \mathrm{~nm}$, the absorption coefficients of rhodopsin and metarhodopsin are equal, $\alpha_{\mathrm{R}}=\alpha_{\mathrm{M}}$. When the quantum efficiencies are also equal, i.e., $\gamma_{\mathrm{R}}=\gamma_{\mathrm{M}}$, then $\beta_{\mathrm{R}}=\beta_{\mathrm{M}}$, and hence in the photosteady state established by $505 \mathrm{~nm}$ light, $f_{\mathrm{M}}(\infty)=0.5$ (Stavenga and Schwemer 1984). In the blue wavelength range, $\beta_{\mathrm{M}} / \beta_{\mathrm{R}}$ is small, and thus illumination with blue light results in a photosteady state with a high metarhodopsin content. In the red wavelength range $\beta_{\mathrm{M}} / \beta_{\mathrm{R}}$ is large, and therefore prolonged red light results in a minimal metarhodopsin fraction, or, a virtually $100 \%$ rhodopsin fraction (Fig. 1a).

The time constant of the photoconversion process,

$\tau=1 /\left(k_{\mathrm{R}}+k_{\mathrm{M}}\right)=1 /\left[I\left(\beta_{\mathrm{R}}+\beta_{\mathrm{M}}\right)\right]$

is inversely proportional to the illumination intensity and the sum of the photosensitivities of $\mathrm{R}$ and $\mathrm{M}$, $\beta_{\text {rel }}=\beta_{\mathrm{R}}+\beta_{\mathrm{M}}$ (Stavenga and Schwemer 1984; Stavenga et al. 2000). $\beta_{\text {rel }}(\lambda)$ is called the relaxation spectrum (Fig. 1a, trace $\beta_{\mathrm{R}}+\beta_{\mathrm{M}}$ ). Its value is virtually constant in the range $490-550 \mathrm{~nm}$, and therefore the time courses of metarhodopsin creation, $f_{\mathrm{M}}(t)$, in Fig. 1b (left) are very similar.

The conversion processes due to various light intensities $I$ applied for a fixed period $t_{0}$ result in metarhodopsin fractions relative to the maximal value (Eq. 1): 

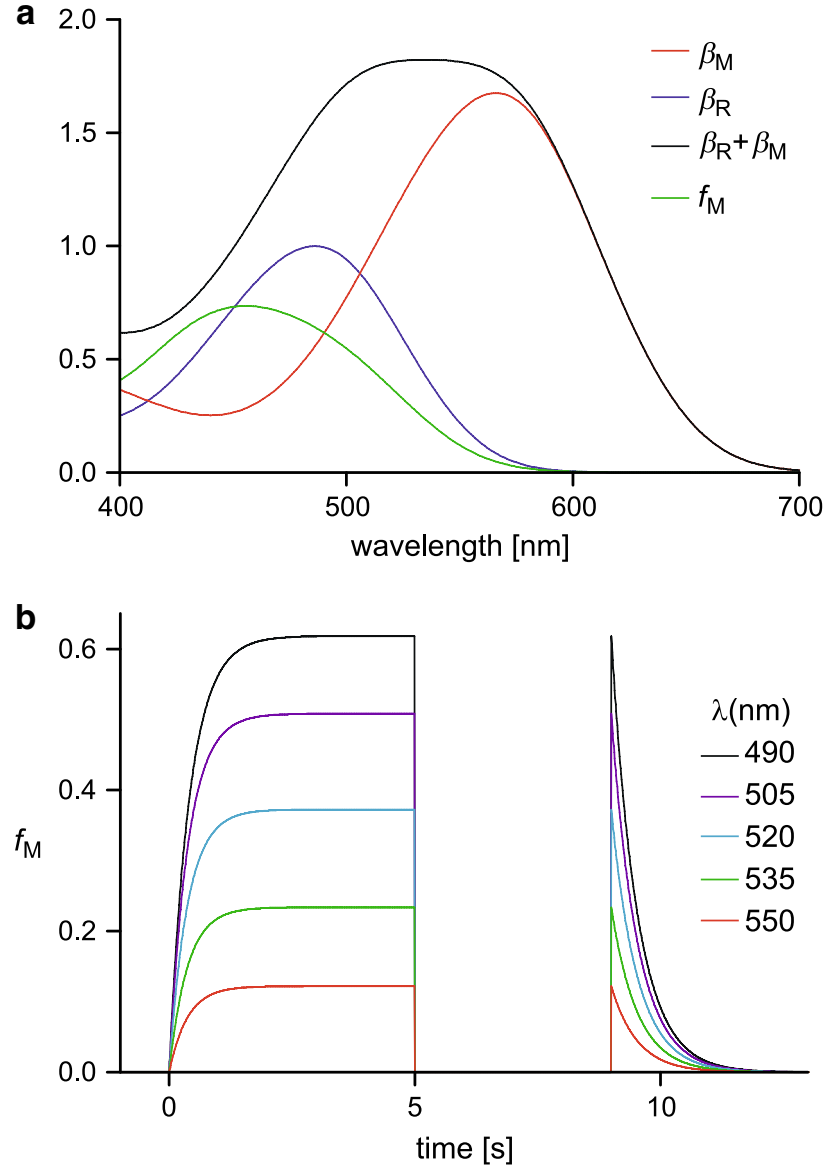

Fig. 1 Spectral properties of the main visual pigment, Rh1, of Drosophila. a The photosensitivities $(\beta)$ of the two thermostable states, rhodopsin (R) and metarhodopsin (M), normalized to the rhodopsin peak, the relaxation spectrum (sum of photosensitivities $\beta_{\mathrm{R}}+\beta_{\mathrm{M}}$ ), and the metarhodopsin fraction in the photosteady state created by monochromatic stimuli with wavelength $\lambda$. The spectra were calculated with the template functions of Govardovskii et al. (2000) using peak wavelength values 486 and $566 \mathrm{~nm}$ for R and M, respectively. b Time course of the metarhodopsin fraction due to irradiating a visual pigment population, where initially all molecules are in the rhodopsin state with monochromatic light $(490-550 \mathrm{~nm}$, $15 \mathrm{~nm}$ steps; left, $5 \mathrm{~s}$ pulse at $t=0 \mathrm{~s}$ ), and the time course of the different reconversions due to subsequent red light of $600 \mathrm{~nm}$ (right, $5 \mathrm{~s}$ pulse at $t=8 \mathrm{~s}$ ). The intensity of the pulses is identical and set so that the time constant of the photoconversion resembles the time constants in the experiment of Fig. 3a. Since the sum of photosensitivities $\beta_{\mathrm{R}}+\beta_{\mathrm{M}}$ hardly changes within the wavelength range presented, the photoconversions show almost identical time courses

$f_{\mathrm{M}}^{*}(I)=f_{\mathrm{M}}(I) / f_{\mathrm{M}}(\infty)=1-\exp (-c / I)$

where $c=t_{0} / \beta_{\text {rel }}$.

When a population of visual pigment molecules has initially a metarhodopsin fraction $f_{\mathrm{M}}(0)$, illumination with red light yields a time course of the metarhodopsin fraction described by

$f_{\mathrm{M}}(t)=f_{\mathrm{M}}(0) \exp \left(-t / \tau_{\mathrm{r}}\right)$ where $\tau_{\mathrm{r}}$ is the time constant for the red light (Fig. 1b, right).

The time course of the metarhodopsin conversions can be quite conveniently studied via fluorescence measurements. Whereas the fluorescence of Drosophila rhodopsin is negligible, the metarhodopsin strongly fluoresces, with a high emission in the red wavelength range (Stavenga 1983; Stavenga et al. 1984). The fluorescence measurements are preferably applied in white-eyed mutants and are performed with a microspectrophotometer where a diaphragm isolates the fluorescence from the deep pseudopupil (Lee et al. 1996; Stark and Thomas 2004). Other pigments than Rh1 metarhodopsin contribute to the emission signal, especially with blue excitation light. A substantial part of the blue-induced fluorescence originates from pigments in the corneal facet lenses and flavoproteins in the mitochondria (Stavenga 1995). Their contribution creates a virtually constant background, on which exponential changes are superimposed, reflecting the visual pigment conversions. The changes in mitochondrial flavoprotein fluorescence, which occur on photoreceptor stimulation (Stavenga 1995), amount to less than $1 \%$ of the fluorescence signal (Zupančič personal communication).

The kinetic scheme of the rhodopsin/arrestin photocycle

There are two different states for both rhodopsin and metarhodopsin, namely the native, active state $R_{a}$, which photoconverts into the active metarhodopsin state $\mathrm{M}_{\mathrm{a}}$, and the inactive states $R_{i}$ and $M_{i}$, which bind arrestin (Fig. 2). Rapidly after the photoconversion of a native rhodopsin molecule to the metarhodopsin state, it binds to arrestin. While the arrestin-metarhodopsin complex is stable, the

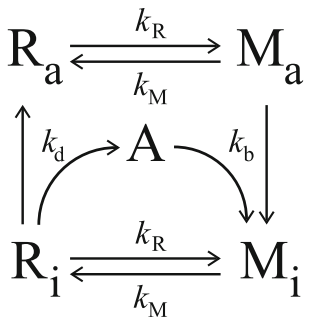

Fig. 2 Simplified diagram of the primary light-induced visual pigment processes in Drosophila. $\mathrm{R}_{\mathrm{a}}$ is the native, active rhodopsin, which on photoconversion yields the active metarhodopsin state, $\mathrm{M}_{\mathrm{a}}$; this state triggers the phototransduction chain resulting in a receptor potential. By phosphorylation and binding of arrestin, $\mathrm{M}_{\mathrm{a}}$ is transformed into the inactive metarhodopsin state, $\mathrm{M}_{\mathrm{i}}$. Photoconversion of $\mathrm{M}_{\mathrm{i}}$ results in the inactive rhodopsin state, $\mathrm{R}_{\mathrm{i}}$, which transforms back to the active rhodopsin state after arrestin release and dephosphorylation (Hardie and Postma 2008). The rate constants for the photoconversions are $k_{\mathrm{R}}$ and $k_{\mathrm{M}}$, and those for arrestin binding and dissociation are $k_{\mathrm{b}}$ and $k_{\mathrm{d}}$, respectively 
rhodopsin-arrestin complex dissociates after several seconds (Fig. 2).

Clearly, the fraction of metarhodopsin molecules in the $M_{i}$ state is limited by the availability of arrestins. The $M_{a}$ state triggers the phototransduction process, and therefore the phototransduction cascade will run continuously when the concentration of metarhodopsin exceeds that of arrestin.

\section{Results}

Visual pigment photochemistry measured via metarhodopsin fluorescence

Using the property of metarhodopsin that it brightly fluoresces under red light excitation, we estimated the relative visual pigment concentration in the intact eyes of two strains of fruitflies, the white-eyed wild type, $w^{-}$, and the white-eyed mutant, $w^{-} ;$arr $2^{3}$. We measured the emission above $695 \mathrm{~nm}$ induced by $600 \mathrm{~nm}$ excitation light. Preadaptation with intense light stimuli at wavelengths between 490 and $600 \mathrm{~nm}$ resulted in various steady-state metarhodopsin fractions, depending on the ratio of the rhodopsin and metarhodopsin photosensitivities (Eq. 2), and subsequent illumination with $600 \mathrm{~nm}$ yielded a decreasing emission due to photoconversion of the previously created metarhodopsin (Fig. 3a).

The final emission value, $B$, is due to background fluorescence emerging from fluorescing non-visual pigments. The difference between the initial and the final value of the red-induced emission, $A(\lambda)$, is proportional to the previously created metarhodopsin fraction, $f_{\mathrm{M}}(\lambda)$; formally, $A(\lambda)=p f_{\mathrm{M}}(\lambda)$. The proportionality constant $p$ depends on the amount of visual pigment contributing to the fluorescence signal, and thus on pigment concentration and measurement aperture. The relative size of the aperture, which slightly varied among the experiments to optimize the signal, was estimated from the background signal $B$. Thus, we have normalized the metarhodopsin-dependent fluorescence signal as $\phi(\lambda)=A(\lambda) / B$ as an estimate for the relative metarhodopsin content in individual specimens of wild-type $w^{-}(n=5)$ and $w^{-} ;$arr $2^{3}$ fruitflies $(n=4)$. The obtained values for $\phi(\lambda)$ indicate that the visual pigment content of the arrestin 2 mutant was about $75 \%$ of the visual pigment content of the wild type (Fig. 3b).

The dependence of the metarhodopsin fraction in the photosteady state on adapting wavelength can be calculated by noting that isosbestic wavelength light $\left(\lambda_{\text {iso }}=505 \mathrm{~nm}\right)$, results in $f_{\mathrm{M}}\left(\lambda_{\text {iso }}\right)=0.5$, so that $A\left(\lambda_{\text {iso }}\right)=0.5 p$. The metarhodopsin fraction created by a pre-adaptation wavelength $\lambda$ therefore can be calculated from $A(\lambda)$ with $f_{\mathrm{M}}(\lambda)=0.5 A(\lambda) / A\left(\lambda_{\text {iso }}\right)$; see Fig. $3 \mathrm{~b}$. The $f_{\mathrm{M}}$ spectra for the
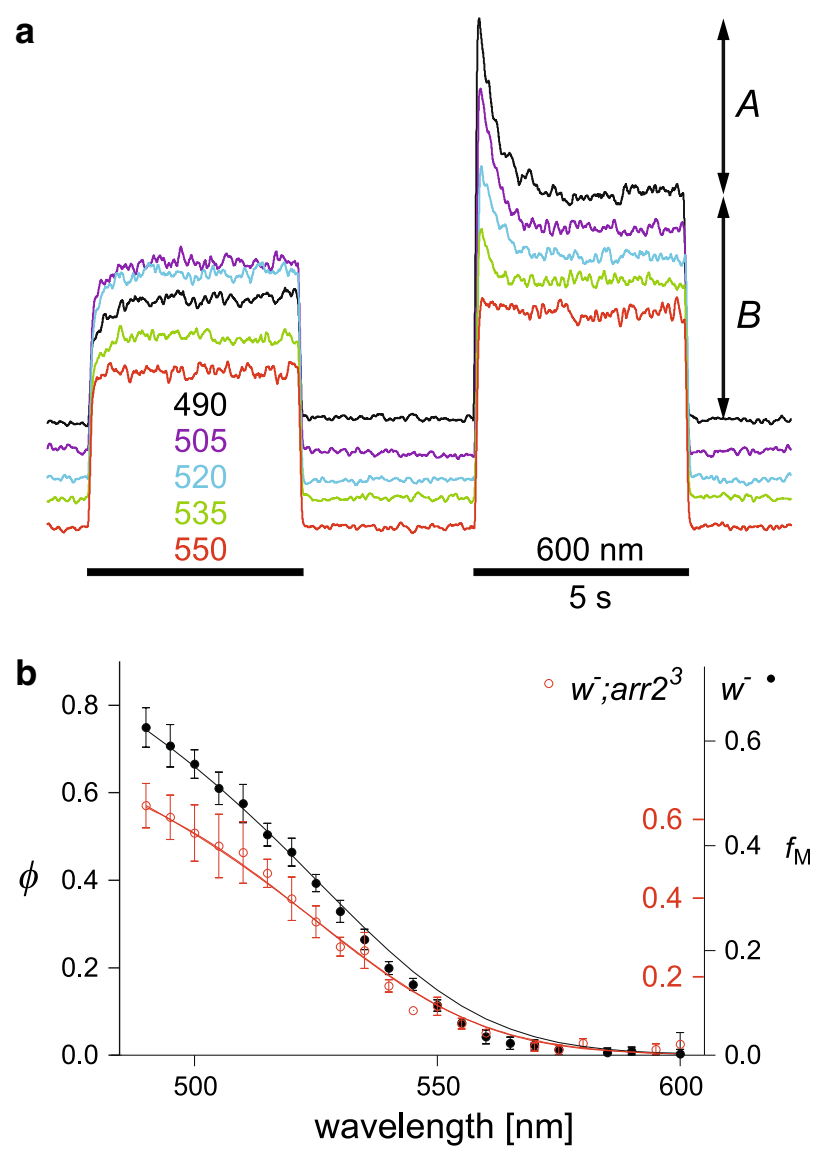

Fig. 3 Metarhodopsin fluorescence as a function of wavelength. a Fluorescence signals induced by a number of monochromatic light pulses (wavelengths $490-550 \mathrm{~nm}$ ) followed by a red $(600 \mathrm{~nm})$ light pulse (Drosophila,$w^{-}$). The values of the initial red-induced emission due to the metarhodopsin, $A$, and the background value, $B$, were measured. $\mathbf{b}$ The ratio $\phi$ of $A$ and $B$ as a function of the wavelength of the adapting light pulse (red symbols $w^{-}$; arr $2^{3}$; black symbols $w^{-}$; error bars SEM). Curves expected for $f_{\mathrm{M}}$ in the photosteady state (Fig. 1a) were fitted to the data, yielding the two right-hand ordinates

wild type and mutant Drosophila then appear to be identical within the measurement error.

ERG and metarhodopsin fluorescence elicited by bright monochromatic light at a range of wavelengths

To correlate the visual pigment conversions with the elicited electrophysiological signal, we measured the ERG simultaneously with the fluorescence, using the same protocol as in Fig. 3a. The ERG responses elicited by the adapting pulses at a range of wavelengths (490-600 nm) were all approximately the same and about maximal (Fig. 4), and the photochemical equilibrium state was always reached.

The ERG during the subsequent darkness (the afterpotential) strongly varied, however, clearly dependent on the amount of metarhodopsin created by the adapting pulse, 

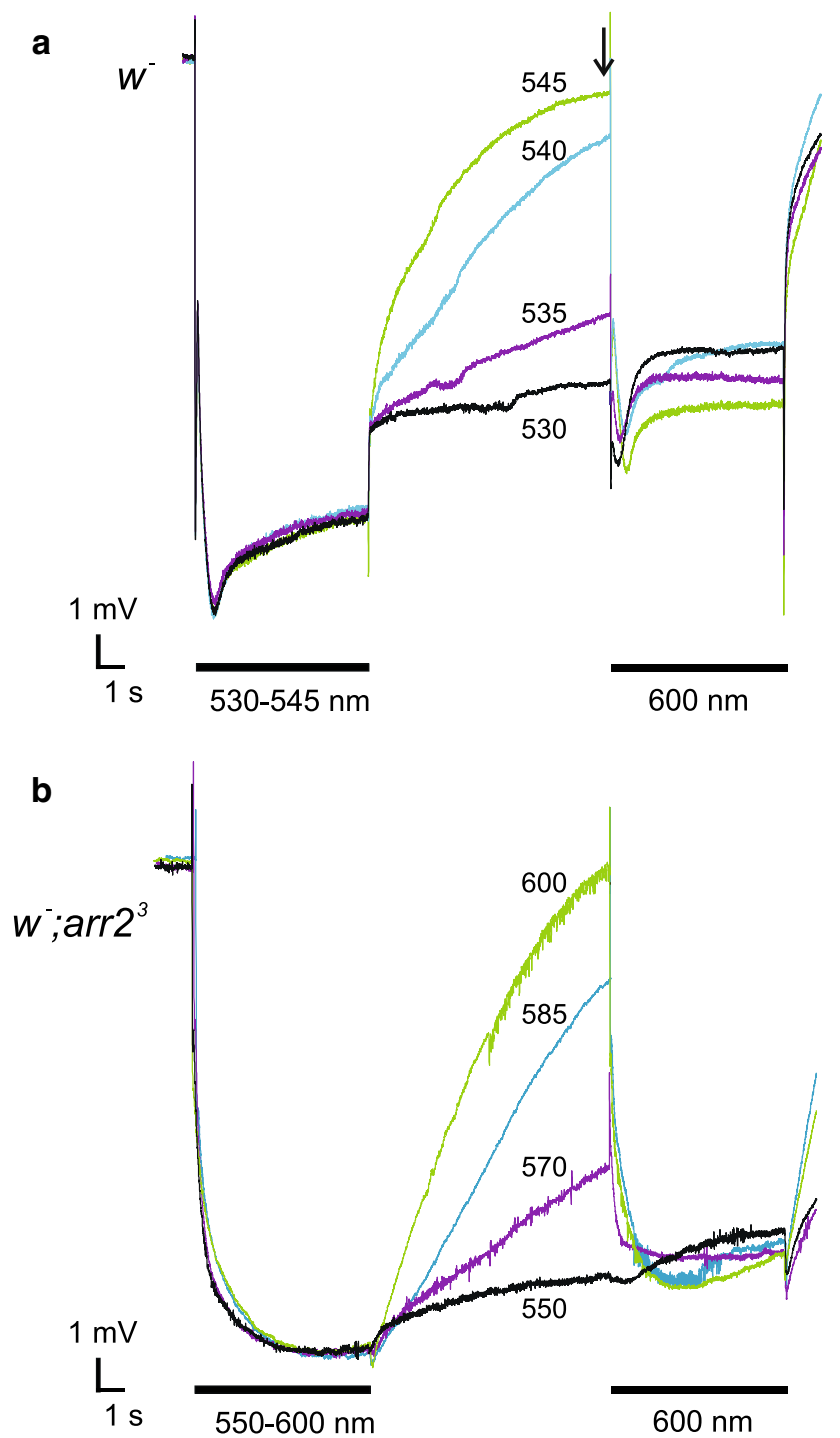

Fig. 4 The ERG during a stimulus sequence similar to that in Fig. 3a measured in (a) the wild type $\left(w^{-}\right)$and (b) the arrestin mutant $\left(w^{-} ; a r r 2^{3}\right)$. A number of monochromatic light pulses, indicated by their peak wavelengths, duration $5 \mathrm{~s}$, were followed by 7-s darkness and a 5-s red $(600 \mathrm{~nm})$ pulse. Note the slow return to the dark level of the ERG in the arrestin mutant. The arrow in a marks the time point at which the afterpotentials were measured

and not on the effective light intensity. Therefore, the experimental paradigm allowed to determine the dependence of the afterpotentials on the metarhodopsin fraction, $f_{\mathrm{M}}$. The response to the adapting pulses at long wavelengths, which created a low metarhodopsin fraction, was followed by a repolarization, i.e., the afterpotential returned to the dark level (Fig. 4a). This no longer occurred after short-wavelength stimuli that created a high $f_{\mathrm{M}}$. The ERG did not repolarize after the response, but was followed by a prolonged depolarizing afterpotential (a PDA), which could be reset with the subsequent red stimulus.
The PDA was quantified by measuring the amplitude of the afterpotential at the end of the dark period, just before the beginning of the red pulse, with respect to the ERG level in the dark prior to the adapting pulse (arrow, Fig. 4a). The time point of measurement, $7 \mathrm{~s}$ after the adapting pulse, allowed for reasonably short experiments, which avoided amplitude changes due to long-term adaptation of photoreceptors during the afterpotential.

Figure 5a shows the normalized amplitudes for both the white-eyed wild-type Drosophila $\left(w^{-}\right.$, Fig. $\left.4 \mathrm{a}\right)$ and the mutant $\left(w^{-} ;\right.$arr $2^{3}$; Fig. $\left.4 \mathrm{~b}\right)$, plotted as a function of the adapting wavelength. The same data are presented in Fig. $5 b$, however, as a function of the created metarhodopsin fraction, derived by using the data of Fig. 3b. The values of the afterpotential amplitude as a function of $f_{\mathrm{M}}$ were fitted with a Hill sigmoid:
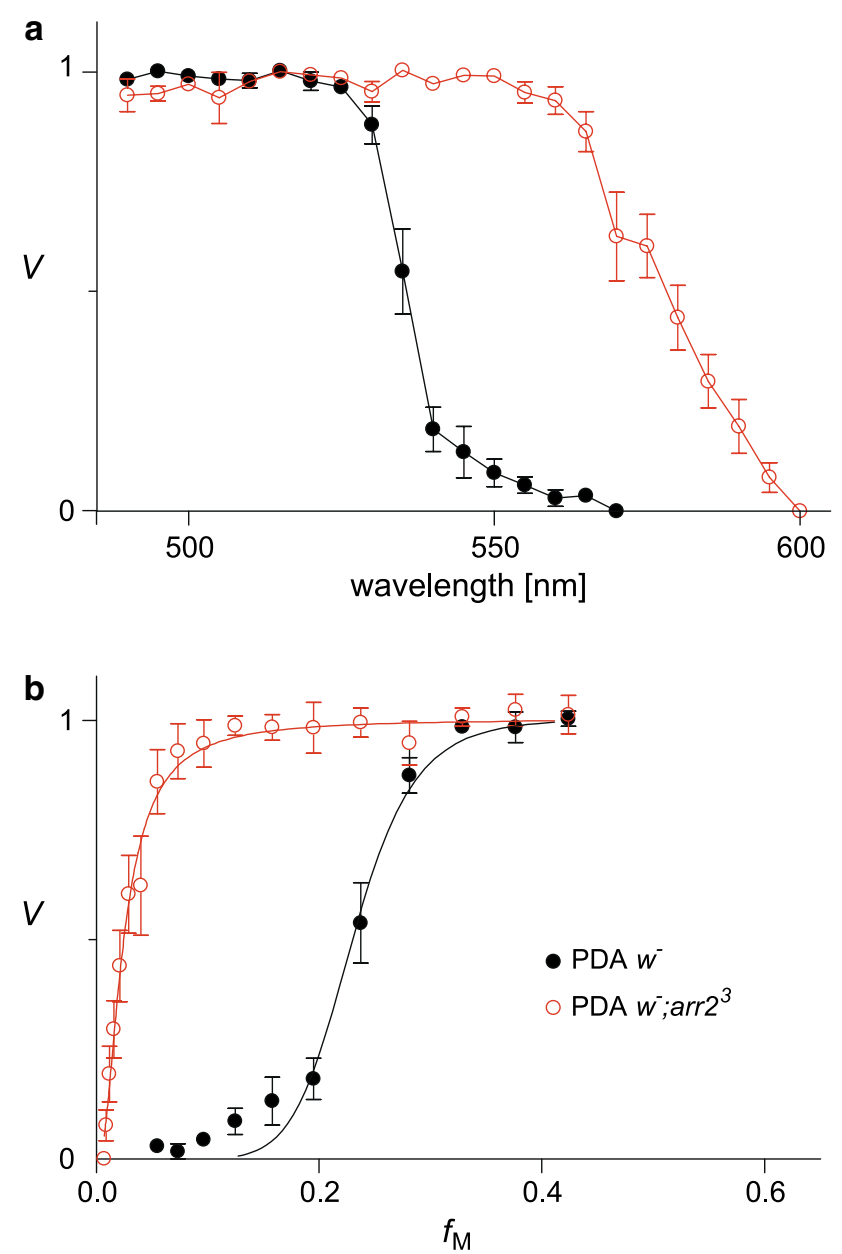

Fig. 5 The dependence of the afterpotential on adapting wavelength and metarhodopsin fraction. a The normalized afterpotential at the end of the 7-s dark period with respect to the ERG level in darkness (see Fig. 4), as a function of the adapting wavelength. b The normalized afterpotential values as a function of the created metarhodopsin fraction, derived by using Fig. 3b, fitted with Hill functions (error bars, SEM) 
$V\left(f_{\mathrm{M}}\right)=f_{\mathrm{M}}^{\mathrm{h}} /\left(f_{\mathrm{M}, 50}^{\mathrm{h}}+f_{\mathrm{M}}^{\mathrm{h}}\right)$

where $f_{\mathrm{M}, 50}$ is the metarhodopsin fraction needed for a halfmaximal response. The transition to the PDA state (10$90 \%$ PDA) occurred at a distinctly higher $f_{\mathrm{M}}$ in the wild type $\left(0.16<f_{\mathrm{M}}<0.29\right)$ than in the arrestin2 mutant $\left(0.01<f_{\mathrm{M}}<0.07\right)$. The $f_{\mathrm{M}}$ values corresponding to a halfmaximal PDA are $f_{\mathrm{M}, 50}=0.23$ for the wild type $\left(w^{-}\right)$and $f_{\mathrm{M}, 50}=0.022$ in the arrestin2 mutant $\left(w^{-} ; a r r 2^{3}\right)$.

ERG and metarhodopsin fluorescence at graded intensities of blue light

The dependence of the electrophysiological response on the adapting light intensity at a fixed wavelength was investigated by applying a graded series of blue stimuli $(490 \mathrm{~nm})$ in both the white-eyed wild type $\left(w^{-}\right)$and the arrestin 2 mutant $\left(w^{-} ; \operatorname{arr} 2^{3} ;\right.$ Fig. 6). To monitor the created metarhodopsin fraction, each blue stimulus (intensity between $-6<\log I<0$ ) was followed by a 5 -s bright red stimulus $(600 \mathrm{~nm})$, which reconverted the metarhodopsin molecules into the rhodopsin state. The metarhodopsin fluorescence signal A (Fig. 6a, b), measured as before, was normalized, plotted as function of $\log I$ (Fig. 7a) and fitted with Eq. 4. At saturation $f_{\mathrm{M}}{ }^{*}=1$, which corresponds to the equilibrium value for $490 \mathrm{~nm}$ light, $f_{\mathrm{M}}=0.62$. The fits for $f_{\mathrm{M}} *(I)$ were identical for both strains within experimental error.
We evaluated the blue light-induced ERG by measuring the response value at the end of the 5-s stimulus with respect to the baseline in the dark (Fig. 7). The slope of the stimulus-response curve in the arrestin2 mutant was steeper and shifted toward lower light intensities (Fig. 7b; $w^{-} ;$arr $2^{3}$, Hill slope $h=0.62, \log I_{50}=-3.44$ ) than in the wild type (Fig. $7 \mathrm{~b} ; w^{-}$, Hill slope $h=0.49, \log I_{50}=$ -3.04 ). This corresponds to an about 2.5 -fold increase in light sensitivity in the mid-intensity range in the mutant.

The stimulus-afterpotential curves (analogous to the stimulus-response curve) of the two strains were spaced further apart (PDA in Fig. 7b; $w^{-}, \log I_{50}=-0.84$; $w^{-} ; \operatorname{arr} 2^{3}, \log I_{50}=-2.2$, a more than 20 -fold intensity difference) than the stimulus-response curves. In the wild type, the intensities to reach half-ERG response and halfPDA were $2.2 \log$ units apart, while in the mutant the difference was $1.2 \log$ units.

Finally, we plotted the dependence of the afterpotential on the metarhodopsin fraction created by the graded intensity blue pulse. We plotted the normalized afterpotential, $V$, as a function of the metarhodopsin fraction, $f_{\mathrm{M}}$. We fitted the data with a Hill function (Eq. 6; Fig. 7c). Here, the $f_{\mathrm{M}, 50}$ values for the two strains also differed strongly $\left(w^{-}: f_{\mathrm{M}, 50}=0.29 ; w^{-} ; a r r 2^{3}: f_{\mathrm{M}, 50}=0.01\right)$.

Kinetic model of the rhodopsin cycle

According to Eq. 1, the normalized metarhodopsin fraction after an illumination period $t_{0}$ is described by $f_{\mathrm{M}}^{*}\left(t_{0}\right)=$
Fig. 6 Simultaneous measurements of the metarhodopsin fluorescence (a, b) and electroretinogram (c, d) from the eyes of a wild-type Drosophila, $w^{-}(\mathbf{a}, \mathbf{c})$ and the arrestin mutant $w^{-} ; \operatorname{arr} 2^{3}(\mathbf{b}, \mathbf{d})$ elicited by monochromatic blue pulses of $490 \mathrm{~nm}$ followed by red pulses $(600 \mathrm{~nm})$. Blue pulses with intensities $\log I>$ -3 created a measurable metarhodopsin fraction as witnessed by the red-induced fluorescence signal (fluorescence decay induced by the $600 \mathrm{~nm}$ pulse) a

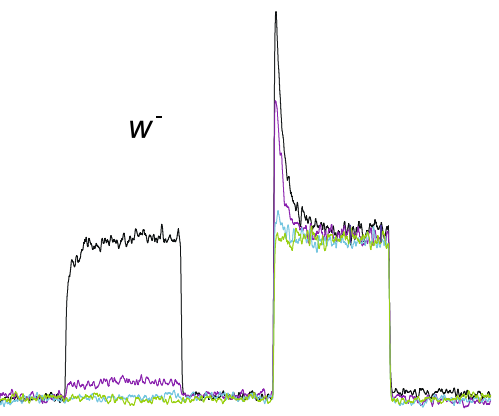

C

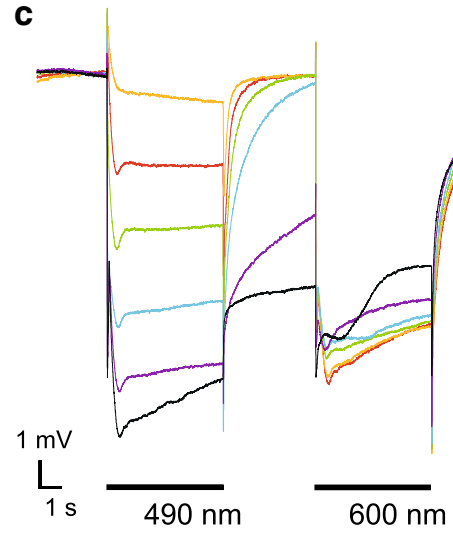

b

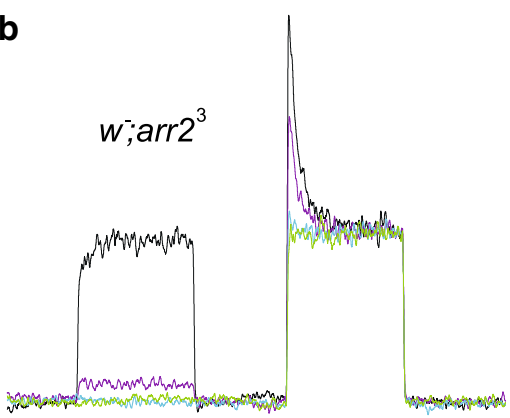

d

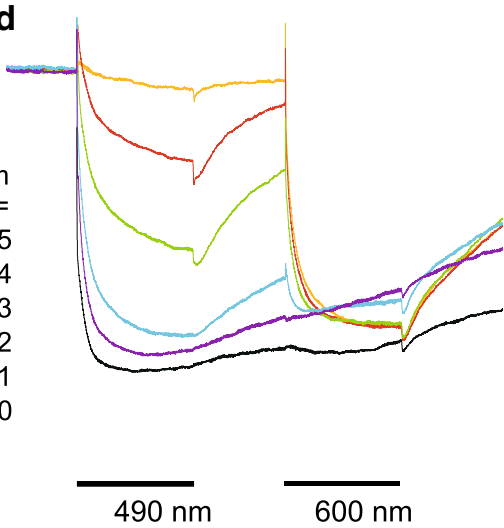



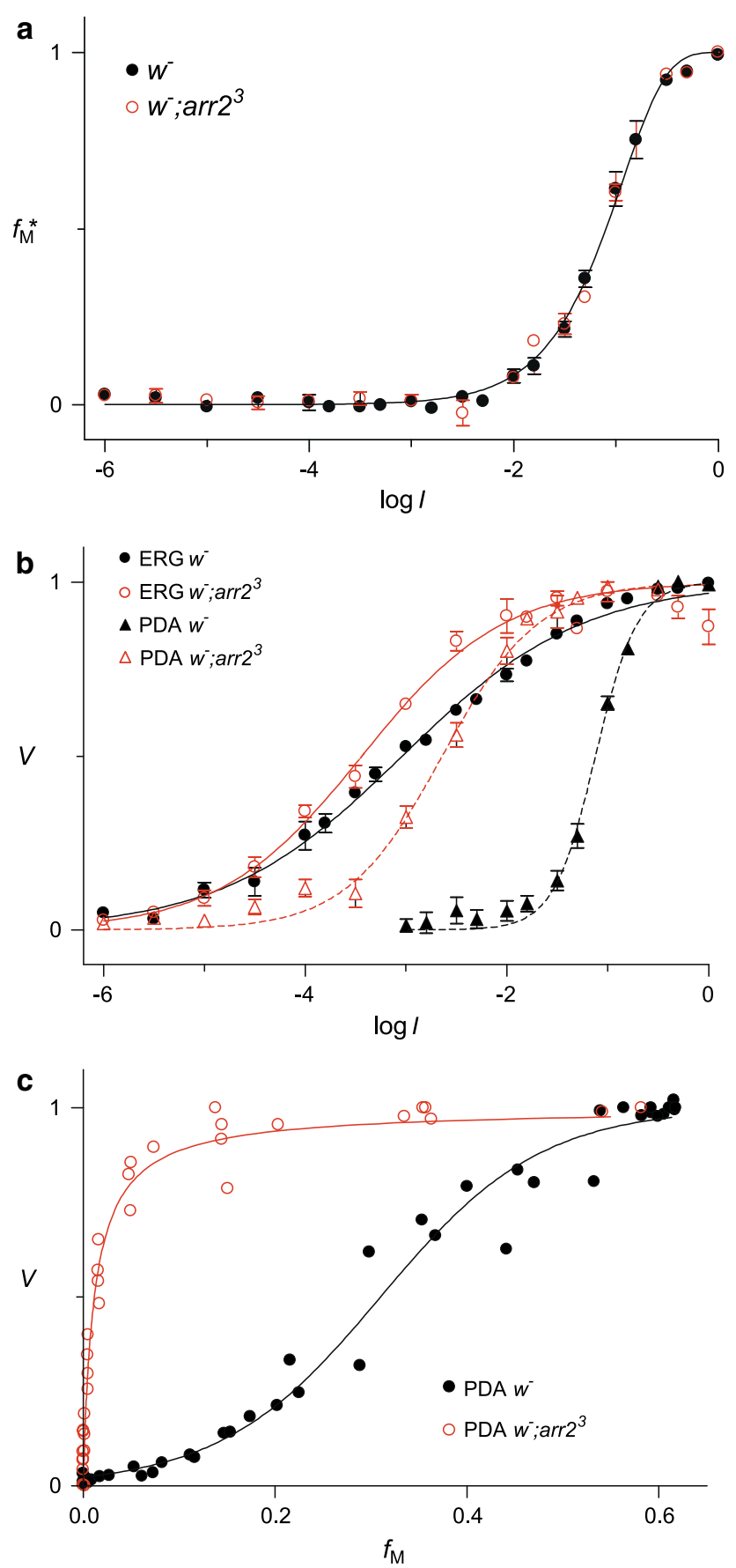

Fig. 7 a Normalized metarhodopsin fraction, $f_{\mathrm{M}}{ }^{*}$, as a function of the intensity of the blue pulse. Data points (error bars, SEM) from both strains virtually coincide and are fitted with a single exponential function of the light intensity, $I$. The fit allows calculation of the $f_{\mathrm{M}}$ in the low light intensity. b Amplitudes $V$ of ERG responses (ERG) and afterpotentials (PDA) elicited by the blue light $(490 \mathrm{~nm})$ pulses of Fig. 6 , for both the wild type $\left(w^{-}\right)$and the arrestin mutant $\left(w^{-} ; \operatorname{arr} 2^{3}\right)$; error bars, SEM. The ERG responses and PDA functions are fitted with Hill functions. $\mathbf{c}$ Afterpotential as a function of $f_{\mathrm{M}}$ created by the graded blue pulses. The $f_{\mathrm{M}}$ value was calculated from the exponential fit in $\mathbf{a}$
$f_{\mathrm{M}}\left(t_{0}\right) / f_{\mathrm{M}}(\infty)=\left[1-\exp \left(-t_{0} / \tau\right)\right]$. With $t_{0}=5 \mathrm{~s}$ and using $\tau=1 /\left(\beta_{\text {rel }} I\right)$ a fit to the fluorescence data of Fig. 7a yielded $\beta_{\text {rel }}=\beta_{\mathrm{R}}+\beta_{\mathrm{M}}=1.73$ (in inverse normalized intensity units). We can use this value to assess the rate of visual pigment conversions associated with the measured ERG values. It follows from the template values (Fig. 1) that for 490-nm light $f_{\mathrm{M}}(\infty)=\beta_{\mathrm{R}} /\left(\beta_{\mathrm{R}}+\beta_{\mathrm{M}}\right)=0.61$, and thus $\beta_{\mathrm{R}}+\beta_{\mathrm{M}}=1.73$ yields $\beta_{\mathrm{R}}=1.05$ and $\beta_{\mathrm{M}}=0.68$. The conversion rate of active rhodopsin to metarhodopsin is given by (Fig. 2):

$\rho=d f\left[R_{\mathrm{a}} \rightarrow M_{\mathrm{a}}\right] / \mathrm{d} t=k_{\mathrm{R}} f_{\mathrm{Ra}}=\beta_{\mathrm{R}} f_{\mathrm{Ra}} I$

where $f_{\mathrm{Ra}}$ is the fraction of visual pigment molecules in the active rhodopsin state. We thus obtain that $\rho=1.05 f_{\mathrm{Ra}} I$. Figure 7 shows for intensities where $\log I<-2, f_{\mathrm{M}} \approx 0$, or $f_{\mathrm{Ra}} \approx 1$, i.e., virtually all visual pigment molecules are in the rhodopsin state. Then $\rho \approx 1.05 I$, or, the $\mathrm{R}_{\mathrm{a}} \rightarrow \mathrm{M}_{\mathrm{a}}$ conversion rate $\rho$ and the applied normalized light intensity $I$ (in inverse normalized intensity units) have about the same numerical value. It thus follows that at $\log I=-3.04$, where the ERG of the wild type $\left(w^{-}\right)$is half-maximal, the $\mathrm{R}_{\mathrm{a}} \rightarrow \mathrm{M}_{\mathrm{a}}$ conversion rate is $9.6 \times 10^{-4} \mathrm{~s}^{-1}$, meaning that in a microvillus with 1,000 visual pigment molecules on average, one $R_{a} \rightarrow M_{a}$ conversion occurs per $1.04 \mathrm{~s}$. In the arrestin2 mutant, a half-maximal response is reached at $\log I=-3.44$ (Fig. 7b), corresponding to an $\mathrm{R}_{\mathrm{a}} \rightarrow \mathrm{M}_{\mathrm{a}}$ conversion rate of $3.86 \times 10^{-4} \mathrm{~s}^{-1}$. The arrestin2 mutant has $75 \%$ of the visual pigment of the wild type and, therefore, in a microvillus with 750 visual pigment molecules, at half-maximal ERG response, one $R_{a} \rightarrow M_{a}$ conversion occurs per $3.5 \mathrm{~s}$.

In the next step, we assessed the active (unarrested) metarhodopsin $\left(\mathrm{M}_{\mathrm{a}}\right)$ concentrations present during the ERG responses of Fig. 6. The created active metarhodopsins are short-lived, because of rapid binding to arrestin, if available. The time constant of metarhodopsin inactivation is $1 / k_{\mathrm{b}}[A]$, where $k_{\mathrm{b}}$ is the binding constant of arrestin to metarhodopsin, and $[A]$ is the arrestin concentration (see Fig. 2). In the wildtype fruitfly, for weak light pulses, this time constant is $23 \mathrm{~ms}$ (Liu et al. 2008), or $k_{\mathrm{b}}[A]=43 \mathrm{~s}^{-1}$. At the end of the $5 \mathrm{~s}$ of illumination applied in our experiments (Figs. 3, 4, 6), a steady state will therefore be fully reached. In the steady state, the active metarhodopsin fraction is constant, or $d f_{\mathrm{Ma}}$ l $d t=k_{\mathrm{R}} f_{\mathrm{Ra}}-k_{\mathrm{b}}[A] f_{\mathrm{Ma}}=0$. At low intensities, where $f_{\mathrm{Ra}} \approx 1$, then $f_{\mathrm{Ma}}=k_{\mathrm{R}} /\left(k_{\mathrm{b}}[A]\right)=\beta_{\mathrm{R}} I /\left(k_{\mathrm{b}}[A]\right)=1.05 I /$ $43=0.024 I$. This means that at an intensity $I=10^{-3.04}$, which creates a half-maximal ERG response in the wild type, $f_{\mathrm{Ma}}=2.2 \times 10^{-5}$. A wild-type Drosophila R1-6 photoreceptor contains 30,000 microvilli with 1,000 visual pigment molecules, or, the number of visual pigment molecules in a photoreceptor is $\sim 3 \times 10^{7}$ (Hardie 2001). At half-maximal 
ERG response, $\sim 2.2 \times 10^{-5} \times 3 \times 10^{7} \approx 660$ molecules of visual pigment, thus, are in the active metarhodopsin state. Assuming a Poisson distribution of photon catch among the microvilli (Hochstrate and Hamdorf 1990), this results in 650 active microvilli at half-maximal depolarization, i.e., of the 650 microvilli, ten received double photon hits.

In the arrestin 2 mutant, the time constant of metarhodopsin inactivation, mediated by arrestin 1 , has not been measured directly, but can be estimated from the current deactivation in isolated $w^{-} ; a r r 2^{3}$ ommatidia, $\tau=167 \mathrm{~ms}$ (Ranganathan and Stevens 1995); or, $k_{\mathrm{b}}[A]=6 \mathrm{~s}^{-1}$. At half-maximal response, where $\log I=-3.44$, we obtain that $f_{\mathrm{Ma}}=\beta_{\mathrm{R}} I /\left(k_{\mathrm{b}}[A]\right)=\beta_{\mathrm{R}} I \tau=1.05 \times 10^{-3.44} \times 0.167=$ $6.1 \times 10^{-5}$. Because the number of visual pigment molecules in the mutant is $\sim 2.25 \times 10^{7}$, the corresponding number of active metarhodopsin molecules at half-maximal depolarization is $\sim 6.1 \times 10^{-5} \times 2.25 \cdot 10^{7} \approx 1,370$, residing in 1,350 active microvilli.

\section{The steady-state PDA model}

According to a deterministic view of the phototransduction process, a single-compartment photoreceptor would abruptly enter a PDA state as soon as the number of metarhodopsin molecules surpassed the number of arrestin molecules. However, our measurements showed that the afterpotential is not a stepwise, but a smooth function of the metarhodopsin fraction (Figs. 5b, 7c). Several factors can be accounted for the smoothing: the stochastics of photon absorption, that of the molecular distribution and the compartmentalization of the photoreceptors into microvilli. To quantitatively investigate the smoothing process, we have developed a steady-state stochastic model, based on binomial statistics, using the same logic as applied to ion channel clutter noise.

We treated four cases with different arrestin concentrations, where the mean number of arrestin molecules per microvillus was $\left\langle N_{\mathrm{A}}\right\rangle=20,100,200$ and 300 , respectively (the bracket notation in this section denotes ensemble means). The cases $\left\langle N_{\mathrm{A}}\right\rangle=20$ and 200 correspond roughly to the $w^{-} ; \operatorname{arr} 2^{3}$ mutant and the wild-type $w^{-}$, respectively. We modeled 5,000 microvilli, with the mean number of visual pigment molecules per microvillus, in all four cases set to $\left\langle N_{\mathrm{P}}\right\rangle=1,000$. To account for the dispersion of microvillar size and other stochastic factors that influence the number of visual pigment and arrestin molecules in each microvillus, both counts were drawn from a Poisson distribution. The count of visual pigment molecules in the metarhodopsin state for each microvillus, $N_{\mathrm{M}}$, was then determined using binomial statistics.

The important parameter for the creation of a PDA is the amount of microvilli where the metarhodopsin molecules outnumber the arrestins, that is the amount of microvilli with a metarhodopsin surplus $\Delta N=N_{\mathrm{M}}-N_{\mathrm{A}}>0$. In the four cases in our model, a microvillus with $N_{\mathrm{P}}=1,000$ visual pigment molecules reached the threshold $\Delta N=0$ at $f_{\mathrm{M}}=0.02, \quad 0.1, \quad 0.2$ and 0.3 , respectively (because $N_{\mathrm{M}}=N_{\mathrm{A}}$ yields $f_{\mathrm{M}}=N_{\mathrm{M}} / N_{\mathrm{P}}=N_{\mathrm{A}} / N_{\mathrm{P}}$ ). The steady-state probability distributions of $\Delta N$ following from our model for the population of 5,000 microvilli are shown in Fig. 8a for two cases where the mean metarhodopsin fraction, $\left\langle f_{\mathrm{M}}\right\rangle=0.02$ and 0.2. Figure $8 \mathrm{~b}$ shows the distributions as a function of $\left\langle f_{\mathrm{M}}\right\rangle$ in the range $0.1-0.4$ for the four cases of $\left\langle N_{\mathrm{A}}\right\rangle$. Figure $8 \mathrm{c}$ presents the fraction of microvilli with a surplus of active metarhodopsin, $P$, as a function of the mean metarhodopsin fraction, $\left\langle f_{\mathrm{M}}\right\rangle$. For instance, when $\left\langle N_{\mathrm{A}}\right\rangle=200$, the transition range is $0.15<f_{\mathrm{M}}<0.25$, and the value $P=0.5$ (half of the microvilli have a metarhodopsin surplus) is reached at $\left\langle f_{\mathrm{M}}\right\rangle \approx 0.2$. We note that all four curves of Fig. 8c could be tightly fitted with a cumulative beta distribution function, a general, twoparameter continuous distribution defined in the range $[0,1]$ (Weisstein 2009).

The metarhodopsin molecules activate the phototransduction process, which results in a receptor potential described by a Hill function. Accordingly, we transformed the $P\left(\left\langle f_{\mathrm{M}}\right\rangle\right)$ function with the sigmoid function

$V(P)=C P^{s} /\left(P^{s}+\left(P_{50}\right)^{s}\right)$

where $s$ is the slope parameter, and $P_{50}$ is the fraction of non-arrested microvilli yielding a half-maximal afterpotential; the normalization constant $C=1+P_{50}^{s}$ is introduced here to achieve $V(1)=1$. We implemented $s=0.5$, a value commonly found for $V(\log I)$ curves in ERG experiments $\left(h=0.49\right.$; Fig. 7b) and $P_{50}=0.022$, because of 650 active microvilli at half-maximal depolarization. The afterpotential function $V\left(\left\langle f_{\mathrm{M}}\right\rangle\right)$ following from Fig. 8c with Eq. 8 could be well fitted with a Hill function (Fig. 8d). We note that the sigmoidally transformed cumulative beta distribution fits were virtually indistinguishable from the Hill function fits (not shown). The model results show that the afterpotential function becomes steeper when the arrestin fraction decreases. The same trend was observed in the experiments with monochromatic light stimuli (Fig. 5b) and varying intensities of $490 \mathrm{~nm}$ light (Fig. 7c). We have included the experimentally obtained $V\left(\left\langle f_{\mathrm{M}}\right\rangle\right)$ curves in Fig. 8d, for direct comparison.

\section{Discussion}

Visual pigment content

We estimated the relative visual pigment content of wildtype flies and arrestin mutants in vivo by measuring the 

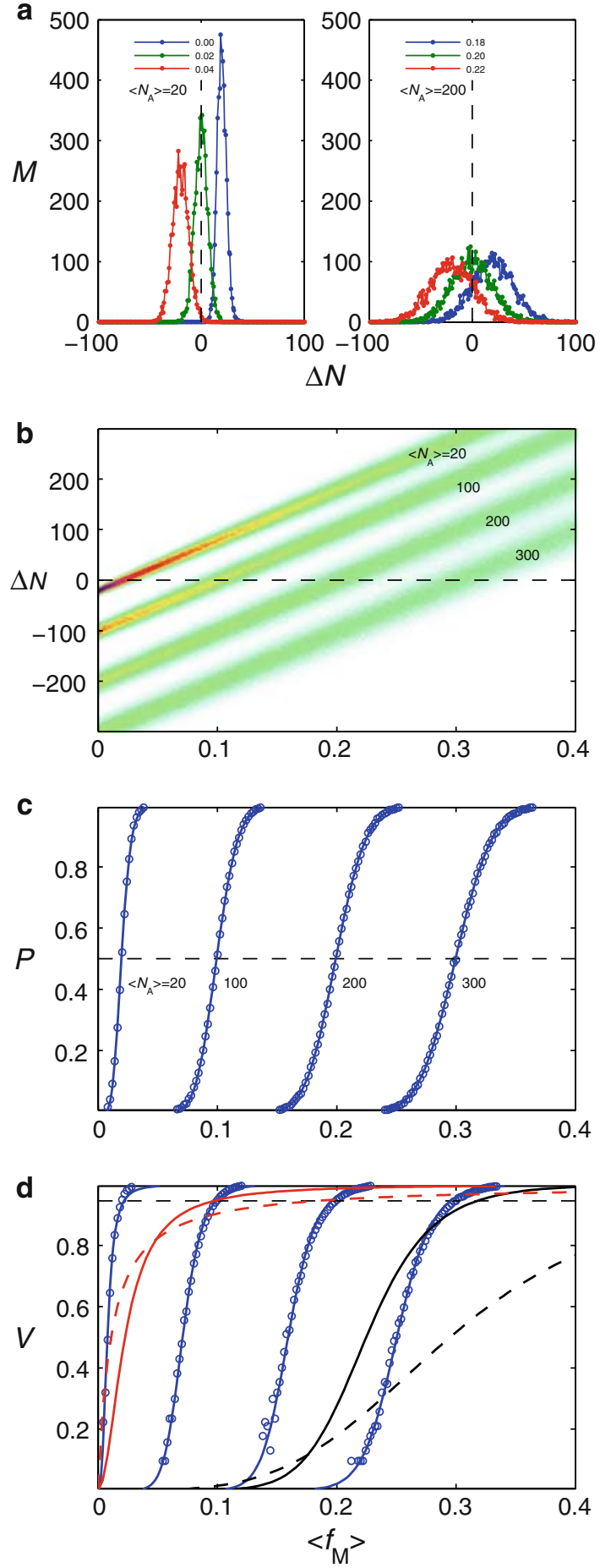

metarhodopsin fluorescence and found that the visual pigment content of the arrestin mutant was about $75 \%$ of that in the wild-type flies (Fig. 3). This difference may be attributed to the age, 1 day $\left(w^{-} ; \operatorname{arr} 2^{3}\right)$ and 5 days $\left(w^{-}\right)$, respectively. Alternatively, the difference may be related to the mutation, because the visual pigment content measured in various Drosophila ninaC mutants with the same fluorescence method yielded distinctly lower visual pigment
Fig. 8 The steady-state PDA transition model and comparison with experimental data. a The number of microvilli, $M$, as a function of the metarhodopsin surplus, $\Delta N$, for a population of 5,000 microvilli with an average 1,000 visual pigment molecules per microvillus. The horizontal bin size is set to 1 . Left histograms for an average number of arrestin molecules per microvillus $\left\langle N_{\mathrm{A}}\right\rangle=20$ and mean metarhodopsin fractions $\left\langle f_{\mathrm{M}}\right\rangle=0.0,0.02$ and 0.04. Right $\left\langle N_{\mathrm{A}}\right\rangle=200$ and $\left\langle f_{\mathrm{M}}\right\rangle=0.18,0.20$ and 0.22 . The area of the histograms with $\Delta N>0$ represents the fraction of microvilli in the PDA state. b Twodimensional histograms of the distribution of $\Delta N$ as a function of $\left\langle f_{\mathrm{M}}\right\rangle$. The four streaks correspond to four cases with average arrestin content $\left\langle N_{\mathrm{A}}\right\rangle=20,100,200$ and 300, respectively. Microvilli with $\Delta N>0$ are in the PDA. Note the narrowing of the histogram at low $\left\langle N_{\mathrm{A}}\right\rangle$. c The PDA function $P\left(\left\langle f_{\mathrm{M}}\right\rangle\right)$. Blue circles show the modeled fractions of microvilli in the PDA state as a function of $\left\langle f_{\mathrm{M}}\right\rangle$. The blue lines are cumulative beta distribution function fits. At $P=0.5$ (horizontal dashed line) half of the microvilli are active. d The afterpotential function $V\left(\left\langle f_{\mathrm{M}}\right\rangle\right)$ obtained by a sigmoidal transformation of $P\left(\left\langle f_{\mathrm{M}}\right\rangle\right)$. The blue curves are Hill fits of $V\left(\left\langle f_{\mathrm{M}}\right\rangle\right)$. The horizontal dashed black line indicates that half of the microvilli are active at about $95 \%$ of the normalized afterpotential. The black and red curves are Hill function fits of the experimental obtained data for $V\left(\left\langle f_{\mathrm{M}}\right\rangle\right)$ in the wild type and arrestin 2 mutant $a r r 2^{3}$, respectively. The solid and dashed curves are the ERG and PDA data taken from Figs. 5b and 7c

contents, depending on the mutant isoform (Hofstee et al. 1996).

\section{Kinetics of visual pigment conversions}

For a receptor with $3 \times 10^{7}$ visual pigment molecules, the $\mathrm{R}_{\mathrm{a}} \rightarrow \mathrm{M}_{\mathrm{a}}$ conversion rate of $\rho=9.6 \times 10^{-4} \mathrm{~s}^{-1}$, derived above for a half-maximal ERG response in the wild type, means per photoreceptor $2.9 \times 10^{4}$ rhodopsin conversions per second. From measurements of quantum bumps with intracellular recordings in R1-6 photoreceptors in whiteeyed Drosophila, Wu and Pak (1978) derived a rhodopsin conversion rate of ca. $10^{4} \mathrm{~s}^{-1}$ at half-maximal depolarization. Our value, obtained from ERG measurements, is thus about a factor 3 higher. The main cause for the discrepancy is probably due to the different electrophysiological methods. While the previous authors recorded from single photoreceptor cells and calibrated their response according to single bumps in dim light, we have recorded from a population of many thousands of photoreceptors, which were not uniformly illuminated, because we used a 0.4 numerical aperture objective, which means that only part of the photoreceptors had their visual field within the illumination aperture, while the rest was stimulated by light scattered in the eye. The stimulus-response curves, thus, were obtained from a large population of non-equally excited photoreceptors. Indeed, while intracellular recordings reveal that Drosophila R1-6 receptors operate over a 4-5 log unit intensity range (Wu and Pak 1978; Juusola and Hardie 2001), we find for the ERG that about 5-6 log units separate the threshold from saturation. 
Arrestin and phototransduction

The ERG stimulus-response curve of the arrestin2 hypomorphic mutant $\operatorname{arr} 2^{3}$ is shifted toward lower intensities over $0.4 \log$ units with respect to the stimulus-response curve of the wild type (Fig. 7). Taking into account the difference in rhodopsin content, this yields a 3.3-fold difference in light sensitivity. Assuming that an ERG response in the midintensity range (around $\log I_{50}$ ) is fully determined by the number of active metarhodopsin molecules in both the wild type and the arrestin mutant, this shift would mean that the $\operatorname{arr} 2^{3}$ mutant has 3.3 times less functional arrestin than the wild type. This value seems to be in conflict with previous biochemical data, which indicated that in the $\operatorname{arr} 2^{3}$ mutant arrestin 2 is reduced 100-fold (Dolph et al. 1993). Considering that in the wild type the arrestin 1 to arrestin 2 ratio is $1: 7$ (Dolph et al. 1993), and that metarhodopsin deactivation by arrestin 1 is less effective than by arrestin2 (Ranganathan and Stevens 1995), the deactivation in $a r r 2^{3}$ should be at least eightfold less effective. Our findings of a 3.3-fold increased light sensitivity in the mutant could therefore indicate that the mutation has been partially compensated by an increased amount of arrestin1.

While the response curve of the arrestin2 mutant is steeper and its midpoint value indicates an increased sensitivity, the mutant and the wild-type responses coincide in the low intensity range, meaning that at low intensities, the photoreceptor sensitivity is arrestin independent. This is in agreement with a recent study by Liu et al. (2008), who demonstrated that in dim light, where sporadic single photon absorptions dominate the receptor potential generation, arrestin will not bind to metarhodopsin unless the latter triggers the signaling cascade.

Arrestin2 binding to metarhodopsin is calcium dependent (Liu et al. 2008). With increasing light intensity, $\left[\mathrm{Ca}^{2+}\right]_{\mathrm{i}}$ increases (Hardie and Postma 2008) and therefore arrestin will progressively reduce phototransduction gain. This feedback action explains the steeper stimulusresponse curve in the arrestin mutant compared to the wild type. The shift of the stimulus response curve of $0.4 \mathrm{log}$ unit is quite moderate, which suggests that the variation in arrestin content only modestly contributes to light adaptation in Drosophila photoreceptors.

Relating the model to the experimental data

We have shown in the model that the functional arrestin fraction, $\left\langle f_{\mathrm{A}}\right\rangle$, numerically coincides with the $\left\langle f_{\mathrm{M}}\right\rangle$ that activates half of the microvilli. As follows from Eq. 8, with $s=0.5$ and $P_{50}=0.022$, half activation of the microvilli $(P=0.5)$ almost saturates the receptor potential at $\sim 95 \%$ of the maximal amplitude. To estimate $\left\langle f_{\mathrm{A}}\right\rangle$ from $\left\langle f_{\mathrm{M}}\right\rangle$ at near saturating levels of the receptor potential with a reverse Hill transformation is, however, quite inaccurate and thus impractical.

We, therefore, applied an alternative approach, which uses the parameter $f_{\mathrm{M}, 50}$, the metarhodopsin fraction needed for a half-maximal response, of the Hill function (Eq. 6) fitted to the $V\left(\left\langle f_{\mathrm{M}}\right\rangle\right)$ curves of Fig. 8d. Figure 9 presents the values of the functional arrestin fraction, $\left\langle f_{\mathrm{A}}\right\rangle$, which correspond to the four modeled $V\left(\left\langle f_{\mathrm{M}}\right\rangle\right)$ curves as a function of the $f_{\mathrm{M}, 50}$-values following from the Hill function fit (open circles) to the modeled data. The relation was well fitted by a power function $\left\langle f_{\mathrm{A}}\right\rangle=\left(f_{\mathrm{M}, 50}\right)^{0.87}$ (Fig. 9). The experiment with the saturating light pulses with different wavelengths (Figs. 5b, 8d) yielded $f_{\mathrm{M}, 50}=0.23$ for the wild type and $f_{\mathrm{M}, 50}=0.022$ for the $w^{-} ;$arr $2^{3}$ mutant. We thus read from Fig. 9 that $\left\langle f_{\mathrm{A}}\right\rangle=0.28$ for the wild type and $\left\langle f_{\mathrm{A}}\right\rangle=0.036$ for the mutant. In other words, the 1,000 visual pigment molecules per microvillus in the wild type are accompanied by about 280 functional arrestins, and the 750 visual pigment molecules per microvillus in the mutant are assisted by 27 functional arrestins. We thus conclude that the arrestin 2 mutant, $\operatorname{arr} 2^{3}$, compared to the wild type, contains about one-tenth of functional arrestin, in line with previous results (Dolph et al. 1993; Ranganathan and Stevens 1995; Vinós et al. 1997).

\section{Comparison of experimental paradigms}

In the present study, we examined three paradigms of fruitfly electroretinography that can be used to assess the rhodopsin-arrestin ratio. In the first paradigm, which is based on the assumption that the light sensitivity is a function of the arrestin content, the photoreceptor

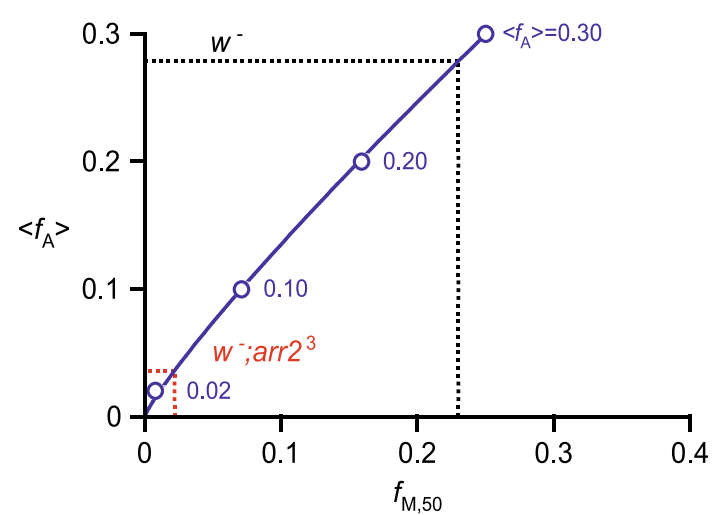

Fig. 9 The arrestin to visual pigment ratio, $\left\langle f_{\mathrm{A}}\right\rangle$, as a function of the metarhodopsin fraction needed for a half-maximal afterpotential, $f_{\mathrm{M}, 50}$. Values obtained from Hill function fits to the modeled afterpotential, $P\left(\left\langle f_{\mathrm{M}}\right\rangle\right)$, curves of Fig. $8 \mathrm{~d}$ are given by open circles, together with a power function fit $\left\langle f_{\mathrm{A}}\right\rangle=\left(f_{\mathrm{M}, 50}\right)^{0.87}$ (blue line). The vertical dashed lines are at the $f_{\mathrm{M}, 50}$ values following from the experimentally obtained curves of Fig. 5b, and the horizontal dashed lines then yield the corresponding $\left\langle f_{\mathrm{A}}\right\rangle$-values (wild type black; arrestin2 mutant; red lines) 
sensitivity is measured. The arrestin content estimated with this method deviated strongly from biochemical data (Dolph et al. 1993). We suggest that this is due to intensitydependent, $\mathrm{Ca}^{2+}$-mediated activation of arrestin, which essentially renders the wild type and the arrestin strain much more alike under dim than under bright illumination. We assume that comparing the light sensitivity of the wild type and the arrestin mutant is not fully reliable, since it does not allow proper control of the rhabdomeric arrestin content during the experiment.

The other two paradigms used were the assessment of the transition to PDA with experimentally set metarhodopsin fractions. The PDA paradigms yielded a rhodopsinarrestin ratio similar to values derived previously (Dolph et al. 1993; Ranganathan and Stevens 1995; Vinós et al. 1997). The transition to PDA was elicited at extreme light intensities, where most of the, if not all available, arrestin was concentrated in the rhabdomere as well as fully activated by $\mathrm{Ca}^{2+}$; its activity remained maximal throughout the entire transition window. Therefore, we suggest that the two PDA paradigms are more reliable than the sensitivity measurement.

The first PDA paradigm where a series of blue pulses of graded intensity was applied, to monitor the transition from full repolarization of the ERG to the PDA, has been extensively used before (Dolph et al. 1993; Ranganathan and Stevens 1995; Vinós et al. 1997), but only in conjunction with the measurement of M-potential (Minke and Kirschfeld 1979). In the second paradigm, the metarhodopsin fraction was set by saturating monochromatic light pulses. Compared to the experimental results of the first paradigm, the results of the second paradigm conform more closely with the predictions of the model, which suggest that the latter method is less sensitive to adverse adaptation and feedback effects. We also note that the second, wavelength paradigm will in general be more easily adapted to non-white-eyed fruitflies and other insect species where measurements of metarhodopsin fluorescence may be cumbersome.

Acknowledgments We thank R. C. Hardie, D. Ready and P. Gonzalez Bellido for useful suggestions and comments. AFOSR/ EOARD provided financial support (Grant no. FA8655-08-1-3012).

Open Access This article is distributed under the terms of the Creative Commons Attribution Noncommercial License which permits any noncommercial use, distribution, and reproduction in any medium, provided the original author(s) and source are credited.

\section{References}

Byk T, Bar-Yaacov M, Doza YN, Minke B, Selinger Z (1993) Regulatory arrestin cycle secures the fidelity and maintenance of the fly photoreceptor cell. Proc Natl Acad Sci USA 90:19071911

Cosens DJ, Briscoe D (1972) A switch phenomenon in the compound eye of the white-eyed mutant of Drosophila melanogaster. J Insect Physiol 18:627-632

Dartnall HJA (1972) Photosensitivity. In: Dartnall HJA (ed) Handbook of sensory physiology, vol VII/1. Springer, Berlin, pp 122145

Dempster J (2001) The laboratory computer: a guide for neuroscientists and physiologists, Academic Press, New York

Dolph PJ, Ranganathan R, Colley NJ, Hardy RW, Socolich M et al (1993) Arrestin function in inactivation of G-Protein coupled receptor rhodopsin in vivo. Science 260:1910-1916

Govardovskii VI, Fyhrquist N, Reuter T, Kuzmin DG, Donner K (2000) In search of the visual pigment template. Vis Neurosci 17:509-528

Hardie RC (2001) Phototransduction in Drosophila melanogaster. J Exp Biol 204:3403-3409

Hardie RC, Postma M (2008) Phototransduction in microvillar photoreceptors of Drosophila and other invertebrates. In: Basbaum AI et al (eds) The senses: a comprehensive reference, vol 1, Vision I. Academic Press, San Diego, pp 77-130

Hardie RC, Raghu P (2001) Visual transduction in Drosophila. Nature 413:186-193

Hochstrate P, Hamdorf K (1990) Microvillar components of light adaptation in blowflies. J Gen Physiol 95:891-910

Hofstee CA, Henderson S, Hardie RC, Stavenga DG (1996) Differential effects of NINAC proteins (p132 and p174) on light- activated currents and pupil mechanism in Drosophila photoreceptors. Vis Neurosci 13:897-906

Juusola M, Hardie RC (2001) Light adaptation in Drosophila photoreceptors: I. Response dynamics and signaling efficiency at $25^{\circ} \mathrm{C}$. J Gen Physiol 117:3-25

Kim JE, Tauber MJ, Mathies RA (2001) Wavelength dependent cistrans isomerization in vision. Biochemistry 40:13774-13778

Lee RD, Thomas CF, Marietta RG, Stark WS (1996) Vitamin A, visual pigments, and visual receptors in Drosophila. Microsc Res Tech 35:418-430

Lee SJ, Xu H, Kang LW, Amzel LM, Montell C (2003) Light adaptation through phosphoinositide-regulated translocation of Drosophila visual arrestin. Neuron 39:121-132

Liu CH, Satoh AK, Postma M, Huang J, Ready DF, Hardie RC (2008) $\mathrm{Ca}^{2+}$-dependent metarhodopsin inactivation mediated by calmodulin and NINAC myosin III. Neuron 59:778-789

Lo MVC, Pak WL (1981) Light-induced pigment granule migration in the retinular cells of Drosophila melanogaster: comparison of wild type with ERG-defective mutants. J Gen Physiol 77:155175

Matsumoto H, Yamada T (1991) Phosrestins I and II: arrestin homologs which undergo differential light-induced phosphorylation in the Drosophila photoreceptor in vivo. Biochem Biophys Res Commun 177:1306-1312

Minke B, Hardie RC (2000) Genetic dissection of Drosophila phototransduction. In: Stavenga DG, DeGrip WJ, Pugh EN Jr (eds) Molecular mechanisms in visual transduction. Handbook of biological physics, vol 3. Elsevier, Amsterdam, pp 449-525

Minke B, Kirschfeld K (1979) The contribution of a sensitizing pigment to the photosensitivity spectra of fly rhodopsin and metarhodopsin. J Gen Physiol 73:517-540

Minke B, Wu CF, Pak WL (1975) Isolation of light-induced response of the central retinula cells from the electroretinogram of Drosophila. J Comp Physiol 98:345-355

Ostroy SE, Wilson M, Pak WL (1974) Drosophila rhodopsin: photochemistry, extraction and differences in the norpA $A^{\mathrm{P} 12}$ phototransduction mutant. Biochem Biophys Res Commun 59:960-966 
Ranganathan R, Stevens CF (1995) Arrestin binding determines the rate of inactivation of the G-protein-coupled receptor rhodopsin in vivo. Cell 81:841-848

Salcedo E, Huber A, Henrich S, Chadwell LV, Chou WH, Paulsen R, Britt SG (1999) Blue- and green-absorbing visual pigments of Drosophila: ectopic expression and physiological characterization of the R8 photoreceptor cell-specific $\mathrm{Rh} 5$ and $\mathrm{Rh} 6$ rhodopsins. J Neurosci 19:10716-10726

Satoh AK, Ready DF (2005) Arrestin1 mediates light-dependent rhodopsin endocytosis and cell survival. Curr Biol 15:1722-1733

Stark WS, Thomas CF (2004) Microscopy of multiple visual receptor types in Drosophila. Mol Vis 10:943-955

Stavenga DG (1983) Fluorescence of blowfly metarhodopsin. Biophys Struct Mech 9:309-317

Stavenga DG (1995) Insect retinal pigments: spectral characteristics and physiological functions. Prog Retin Eye Res 15:231-259

Stavenga DG (2002) Colour in the eyes of insects. J Comp Physiol A 188:337-348
Stavenga DG, Schwemer J (1984) Visual pigments of invertebrates. In: Ali MA (ed) Photoreception and vision of invertebrates. Plenum, New York, pp 11-61

Stavenga DG, Franceschini N, Kirschfeld K (1984) Fluorescence of housefly visual pigment. Photochem Photobiol 40:653-659

Stavenga DG, Oberwinkler J, Postma M (2000) Modeling primary visual processes in insect photoreceptors. In: Stavenga DG, DeGrip WJ, Pugh EN Jr (eds) Molecular mechanisms in visual transduction. Handbook of biological physics, vol 3. Elsevier, Amsterdam, pp 527-574

Vinós J, Jalink K, Hardy RW, Britt SG, Zuker CS (1997) A G proteincoupled receptor phosphatase required for rhodopsin function. Science 277:687-690

Weisstein EW (2009) Beta distribution. From Mathworld-A wolfram web resource. http://mathworld.wolfram.com/Beta Distribution.html

Wu CF, Pak WL (1978) Light-induced voltage noise in photoreceptor of Drosophila melanogaster. J Gen Physiol 3:249-268 